Pensamiento Crítico N. ${ }^{\circ}$ 15, pp. 129-138

\title{
Los fundamentos teóricos de los tratados de libre comercio
}

\author{
Pablo Rivas Santos
}

\section{RESUMEN}

El presente artículo evalúa los fundamentos teóricos de los tratados de libre comercio considerando los efectos del comercio entre países (dotadas desigualmente por la naturaleza) suponiendo que las respectivas producciones pueden libremente ser transportadas de un país a otro; pero no los trabajadores ni los bienes de capital acumulados.

La división del trabajo entre países incrementa la productividad del esfuerzo laboral, por lo que resulta ventajosa para los países, pese a que las condiciones internas de producción puedan ser más favorables a un país A que a otro país $\mathrm{B}$. Conviene que el país A (país mejor dotado material y tecnológicamente) produzca bienes en el que sea mayor su superioridad dejando al país B (país menor dotado de material y tecnología) que produzca bienes en la que la superioridad del país A sea menor.

Esa incongruencia de no explotar esas condiciones internas de producción más favorables, yendo a comprar esos bienes (que podrían producirse en el país A) en el país $\mathrm{B}$ cuyas condiciones de producción son más desfavorables, se origina por la inmovilidad de los factores trabajo y capital, que no pueden acudir al país A de producción más favorable. 


\section{Pensamiento Crítico N. 15}

La ley de asociación evidencia por qué hay una tendencia a ir gradualmente intensificando la cooperación entre países. La ley de asociación es el móvil que continuamente estimula a los países a unirse, en busca de mutua cooperación. Todo progreso hacia una más avanzada división del trabajo favorece los intereses de los países que participan en dicha división. El actuar de la gente, estimulado por la percepción de la mayor productividad del trabajo bajo la división del mismo, genera un mayor comercio entre países y los hace desarrollarse progresivamente.

La economía internacional evidencia cuáles son los factores que provocan la asociación y su progresivo desarrollo. Mientras el trabajo sea más fructífero bajo el signo de la división del trabajo y en tanto la gente sea capaz de advertir tal realidad, el actuar de la gente tenderá espontáneamente a la cooperación y asociación. Esa condición -la mayor productividad de la división del trabajo- aparece debido a una realidad: la innata desigualdad de los hombres y la desigual distribución geográfica de los factores naturales de producción.

Palabras clave: División del trabajo, ley de asociación, productividad del trabajo, capital.

\section{ABSTRACT}

This article evaluates the theoretical foundations of free trade agreements considering the effects of trade between countries (unequally endowed by nature) when it is assumed that the respective productions can be transported freely from one country to another, but not the workers or the capital equipment.

The division of labor between countries, increase productivity and work effort, therefore, advantageous to countries, despite the internal conditions of production may be more favorable to country A than country B. Should country A (country better equipped and technologically material) to produce goods in which its superiority is greater leaving the country $\mathrm{B}$ (country worst equipped and technologically material) to produce goods in which the superiority of country A is less.

This inconsistency does not exploit these internal conditions more favorable production, going to buy these goods (which could occur in country A) in country $\mathrm{B}$ in which production conditions are more unfavorable results from the immobility of labor and capital, who can not go to country more favorable production. 


\section{Pablo Rivas Santos}

The law of association clear why there is a tendency to gradually strengthen cooperation between countries. The law of association is the motive to continue to encourage countries to join in search of mutual cooperation. Any progress towards a more advanced division of labor in the interests of the countries participating in the division of labor. The act of the people, stimulated by the perception of increased productivity of labor under the division of labor generates more trade between countries and makes them progressively developed

The International Economy evidence the factors that cause the association and its progressive development. While the work is more productive under the sign of the division of labor and as long as people are able to realize this reality, the people tend to act spontaneously to cooperation and partnership. That condition, the higher productivity of the division of labor appears because their cause is a reality: the innate inequality of men and the uneven geographical distribution of natural factors of production.

Keywords: Division of labor, partnership act, labour productivity, capital.

\section{Introducción}

La sociedad brinda a la gente medios excepcionales para alcanzar todas sus metas. Mantener la sociedad es el supuesto esencial de toda actuación que pretenda llevar a buen fin.

La mayor productividad de la cooperación social ha hecho que la población se incremente en mayor número. La gente coopera bajo la división del trabajo porque así atienden mejor sus propios intereses.

La sociedad implica acción mancomunada y cooperativa, en la que cada persona considera el provecho ajeno como medio para alcanzar el propio. La sociedad es acción y reacción mutua; interacción -influencia recíproca- entre todos los países; y acción cooperativa; con miras a que los participantes puedan alcanzar sus propios fines. 


\section{Pensamiento Crítico N. ${ }^{\circ}$}

El hombre ordena su conducta con racional deliberación. El hombre, para no perturbar el mecanismo de la cooperación social, se abstiene de dar satisfacción a aquellos deseos que impedirían el mantenimiento de las instituciones sociales. Prefiere dejar insatisfechos ciertos deseos incompatibles con la vida social; para satisfacer otros deseos que sólo de modo más perfecto pueden ser atendidos bajo el signo de la división del trabajo. Así, emprende el camino que conduce a la cooperación social y la riqueza.

Las diferencias entre la gente son resultado de la educación, oportunidades personales y las instituciones sociales. La cooperación social y la división social del trabajo (que se perfecciona entre gente más eficiente en cualquier sentido; y gente menos eficiente en cualquier sentido) beneficia a todos los participantes. La Economía se opone a la concesión de privilegios personales; porque restringe la prosperidad de la gente. Pregona la igualdad de todos ante la ley porque tal política beneficia a la comunidad. Afirma que no existe igualdad entre los hombres. Afirma la conveniencia social que encierra la democracia, propiedad privada y la libertad.

La gente debe tomar en cuenta la idea que pregona: la armonía existente entre los intereses correctamente entendidos de todos, ya se trate de individuos, grupos sociales o países. La gente se da cuenta que recurriendo a la cooperación social y división del trabajo cuida mejor su bienestar personal. Sólo en un ambiente de paz cabe recurrir a la cooperación social y división del trabajo.

\section{División social del trabajo}

La división del trabajo (con su efecto, la cooperación) es un fenómeno social. La experiencia enseña que la acción mancomunada es más eficaz y de mayor productividad que la acción individual. Las circunstancias naturales que estructuran la vida y el esfuerzo

de la gente hacen que la división del trabajo incremente la productividad por unidad de esfuerzo invertido.

Circunstancias naturales que provocan la aparición de la división del trabajo:

1) La innata desigualdad de la capacidad de la gente para hacer específicos trabajos.

2) La desigual distribución de los recursos naturales sobre la tierra. 


\section{Pablo Rivas Santos}

3) Hay obras cuya ejecución excede la capacidad laboral de un solo individuo, exigiendo la unión de esfuerzos. Hay obras que podrían ser realizadas por un individuo aislado; pero su duración sería tan dilatada que retrasaría excesivamente el disfrute de esas obras y no compensaría, la labor realizada. En ambos casos, el esfuerzo humano mancomunado permite alcanzar el objetivo deseado.

El incremento de la productividad (típico de la división del trabajo) se registra, siempre que la desigualdad sea tal que cada individuo o cada parcela de tierra sea superior (por lo menos en algún aspecto) a los demás individuos o parcelas. Por ejemplo, si Japón produce 6 artefactos o 4 camisas y Perú produce 2 camisas (si bien Perú puede producir hasta 8 camisas); bajo el signo de la división del trabajo, dedicándose Perú y Japón, únicamente, a aquella labor en que mayor sea su respectiva eficiencia, en total producirán 6 artefactos +8 camisas.

\section{Ley de asociación}

La ley de asociación evidencia los efectos de la división del trabajo. Ejemplo, cuando la industria japonesa colabora con la industria peruana siendo la industria japonesa de mayor eficiencia, en cualquier aspecto, que la industria peruana.

Investigando los efectos del comercio entre países (dotados desigualmente por la naturaleza), suponiendo que las respectivas producciones pueden libremente ser transportadas de un país a otro; pero no los trabajadores ni los bienes de capital acumulados.

La división del trabajo entre países incrementa la productividad del esfuerzo laboral, por tanto, resulta ventajosa para los países, pese a que las condiciones internas de producción puedan ser más favorables a un país $\mathrm{A}$ que a otro país $\mathrm{B}$. Conviene que el país $\mathrm{A}$ (país mejor dotado material y tecnológicamente) produzca bienes en el que sea mayor su superioridad dejando al país B (país peor dotado material y tecnológicamente) que produzca bienes en la que la superioridad del país A sea menor.

Esa incongruencia de no explotar esas condiciones internas de producción más favorables, yendo a comprar esos bienes (que podrían producirse en el país A) en el país $\mathrm{B}$, cuyas condiciones de producción son más desfavorables, se origina por la inmovilidad del trabajo y capital, que no pueden acudir al país A de condición más favorable. 


\section{Pensamiento Crítico N. 15}

La ley de costos comparativos -que aborda el problema que causa el comercio internacional- es un caso de la ley de asociación.

Ejemplo aclaratorio. Si Japón goza de mayor eficiencia que Perú; tal que para producir un polo, Japón necesita 3 horas y Perú necesita 5 horas; y para producir un artefacto Japón necesita 2 horas y Perú necesita 4 horas; resulta que ambos ganan si Japón produce artefactos y Perú produce polos.

Si Perú y Japón dedican 60 horas para producir polos y 60 horas para producir artefactos. Si Japón solo produce artefactos, produciría 60 artefactos en 120 horas; Perú, en el mismo supuesto, si solo produce polos, produciría 24 polos en 120 horas. La suma de sus actividades será 60 artefactos +24 polos; ya que los polos tienen para Japón un cociente de sustitución de 3/2 artefacto y para Perú de 5/4 artefacto. Por tanto, la división del trabajo beneficia a Perú y Japón. La colaboración de aquellos de más talento, habilidad y destreza con aquellos peor dotados material y tecnológicamente es ventajosa para ambos países. Las ganancias derivadas de la división del trabajo son recíprocas.

La ley de asociación evidencia por qué hay una tendencia a ir gradualmente intensificando la cooperación entre países. La ley de asociación es el móvil que continuamente estimula a los países a unirse, en busca de mutua cooperación. Todo progreso hacia una más avanzada división del trabajo favorece los intereses de los países que participan en la división del trabajo. El actuar de la gente, estimulado por la percepción de la mayor productividad del trabajo bajo la división del trabajo, está generando un mayor comercio entre países y los hace progresivamente desarrollarse.

La economía internacional evidencia cuáles son los factores que provocan la asociación y su progresivo desarrollo. Mientras el trabajo sea más fructífero bajo el signo de la división del trabajo, y en tanto la gente sea capaz de advertir tal realidad, el actuar de la gente tenderá espontáneamente a la cooperación y asociación. Esa condición -la mayor productividad de la división del trabajo- aparece porque su causa viene de una realidad: la innata desigualdad de los hombres y la desigual distribución geográfica de los factores naturales de producción. 


\section{Pablo Rivas Santos}

\section{Análisis de la ley de asociación}

La ley de costos comparativos es una amenaza para los planes de quienes pretenden justificar el proteccionismo, para privilegiar los intereses de algunos empresarios. El objetivo de formular esta ley es refutar un cuestionamiento al libre comercio internacional. El proteccionista se pregunta: bajo el libre comercio, ¿̇cuál será el destino del Perú, cuyas condiciones de producción son más desfavorables que las condiciones de producción de cualquier país desarrollado?

En un mundo globalizado donde capital, trabajo y productos gozan de plena libertad de movimiento, aquel país (poco idóneo para la producción) dejaría de ser un lugar atractivo para la inversión. En tal caso, si los países desarrollados satisfacen mejor sus necesidades no explotando las condiciones comparativamente más imperfectas que ofrecía el país en desarrollo, no invertirán en aquel país. Pero David Ricardo quiso enfrentarse con los problemas reales que origina el mundo, en que las circunstancias específicas de cada caso vienen predeterminadas por los países formados en épocas anteriores donde capital y trabajo se hallan ligados al suelo por diversas razones de orden institucional. En tales circunstancias, el libre comercio (la libertad de movimientos limitada solo a los productos) no provocaría la distribución del capital y trabajo, sobre la tierra; según las posibilidades, mejores o peores, que cada lugar ofrezca en relación a la productividad del esfuerzo humano. Entonces entra a tallar la ley de costos comparativos. Cada país produce para las que sus específicas condiciones le ofrecen relativa (aunque no las mejores) oportunidades. Para los japoneses es más ventajoso dejar de explotar sus capacidades para hacer polos, pese a ser estas capacidades superiores a las capacidades para hacer polos en Perú, importando en su lugar los polos producidos en Perú en condiciones más desfavorables ${ }^{1}$.

En la conclusión, en el teorema de costos comparativos están implícitos dos supuestos:

- La productividad de los factores de producción técnicamente posibles de trasladar es diferente según los lugares donde se ubiquen.

- Esos factores por razones institucionales tienen restringida su movilidad.

1 Se trata de un caso análogo al del dentista, que para elaborar planchas contrata los servicios de un mecánico dental, no obstante superarle también en ese específico cometido, para dedicarse exclusivamente a la curación, en la que su ventaja es todavía más notoria. 


\section{Pensamiento Crítico N. I5}

En la ley de costos comparativos comparamos la producción de dos bienes distintos. Si tal comparación es factible es porque suponemos que para producir cada bien distinto (aparte de un factor específico) sólo se requieren factores no específicos de la misma clase.

Sólo las diferentes condiciones institucionales, que restringen la movilidad de mercancías y factores de producción, hacen a la gente distinguir el mercado nacional del mercado internacional.

Si no se quiere estudiar la ley de costos comparativos es obligado ir al cálculo monetario ${ }^{2}$.

Si las circunstancias son tales que en EE.UU. producir una camisa a y un jarabe $b$ necesita 1 jornada de la misma clase de trabajo; mientras que Perú (con la misma inversión de capital) necesita 2 jornadas para a y 3 jornadas para $b$; siendo ese capital y $a, b$ libremente transferibles de EE.UU. a Perú y viceversa, pero no la mano de obra. Los salarios en Perú, para producir a son la mitad de los salarios americanos y para producir $b$, la tercera parte.

Si el jornal americano es 6 dólares, en Perú será de 3 dólares en producir a y de 2 dólares en producir $b$. Tal diferencia en la remuneración de trabajo de la misma clase no puede perdurar; si en el mercado interno de Perú la mano de obra goza de movilidad: los obreros abandonarán la producción de $b$, enrolándose a la producción de $a$; este movimiento hará que baje la remuneración en $a$, y suba en $b$. Finalmente, los salarios peruanos, se igualarían en ambas industrias. Aparecería entonces una tendencia a ampliar la producción de a y a desplazar a la competencia americana. Por otra parte, la producción de $b$ en Perú, dejaría de ser rentable, lo que obligaría a abandonar esa producción de $b$, mientras en EE.UU. se incrementaría esa producción de $b^{3}$.

2 Tomando al ejemplo de un dentista y su mecánico dental: si el dentista puede emplear su limitada capacidad de trabajo en hacer curaciones que le dan ingresos de 50 dólares la hora, le convendrá contratar los servicios de un mecánico dental que le haga los moldes, pagándole 2 dólares la hora, aun cuando el mecánico dental emplee 3 horas para realizar lo que el cirujano podría hacer en 1 hora.

3 Se llega a la misma conclusión suponiendo que la diferencia en las condiciones de producción depende, parcial o exclusivamente, en la distinta cantidad de capital que, en cada caso, fuera necesario invertir. 


\section{Pablo Rivas Santos}

\section{Conclusiones}

La división del trabajo es el efecto provocado por consciente reacción de la gente ante la desigualdad de las circunstancias naturales del mundo. Además, la propia división del trabajo incrementa esa desigualdad. A causa de ella, las diversas zonas geográficas asumen funciones específicas en el complejo proceso de producción. Debido a esa diversidad, determinadas zonas: se convierten en urbanas y otras en rurales; y se ubican en diferentes lugares las distintas ramas de la industria, minería y agricultura. Mayor importancia aún tiene la división del trabajo en relación a aumentar la innata desigualdad humana: la práctica y dedicación a tareas específicas adapta, cada vez en mayor grado, a los interesados en las correspondientes exigencias; la gente desarrolla más algunas de sus facultades innatas, descuidando otras. Surgen las personas con vocación, las personas se especializan.

La división del trabajo descompone los diversos procesos de producción en mínimas tareas, muchas de las cuales pueden ser realizadas mediante dispositivos mecánicos electrónicos. Tal circunstancia permite recurrir a la máquina electrónica, lo cual provoca gran progreso en los métodos técnicos de producción. La mecatrónica es consecuencia de la división del trabajo. La maquinaria electrónica se instala en un ambiente social donde impera la división del trabajo. Todo nuevo progreso en el uso de maquinaria más precisa, refinada y productiva exige una mayor especialización de cometidos. 


\section{Pensamiento Crítico N. ${ }^{\circ}$}

\section{Bibliografía}

BARRO, Robert J. (1990). Macroeconomía. Madrid: Alianza Editorial.

FRIEDMAN, Milton (1976). Teoría de precios. Madrid: Alianza Editorial.

MUNDELL, Robert A. (1978). El hombre y la economía. Madrid: Amorrous Editores.

ROPCKE, Wilhelm (1967). Economía política. Madrid: Alianza Editorial.

ROTHBARD, Murray N. (1980). Moneda libre y controlada: Ensayo sobre el origen y función de la moneda. Buenos aires: Centro de Estudios sobre la Libertad.

VON HAYEK, Friedrich (1960). Precios y producción. Mc Graw Hill.

VON MISES, Ludwig (1981). Acción humana. Valencia: Fundación Ignacio Villalonga. 